\title{
Water Extract of Dragon Fruit Peel Catalyzed Synthesis of Dihydropyridines By Hantzsch Condensation
}

\author{
Abdulfatah Abdullah Abdu Saifan*1 and Sultan Abduh Al-horaibi*2 \\ ${ }^{1}$ School of Chemical Sciences, S.R.T.M University, Nanded, India \\ ${ }^{2}$ Department of Chemistry, Al-Baydah University, Yemen \\ *Corresponding Author: Email abdulfatahsaifan123@gmail.com
}

\begin{tabular}{llllll}
\hline Received: 29.03.2021 $\quad$ Accepted: 03.04.2021 & • & Published: 30.04.2021 & • Final Version: 30.04.2021
\end{tabular}

\begin{abstract}
Nowadays, natural substances are increasingly used in organic synthesis for their safety aspects towards the environment. That is why we identified a natural substance through which it promoted the organic reaction, and it could be sustainable and from plant sources obtained, and through this natural substance, it promotes the synthesis of dihydropyridine and its derivatives under solvent-free conditions. We have resorted to this method, being economical, free of minerals and solvents, to create highly functional dihydropyridine derivatives. It is promoted by the water extract of dragon Fruit (WED) at a temperature of $\left(80^{\circ} \mathrm{C}\right)$. These (WED) promoted reactions are found to afford high yield for the desired products and this method is Protocol an alternative to the current procedures.
\end{abstract}

Keywords: Dihydropyridine derivatives; Greener synthesis; Nature derived catalyst; Water extract of dragon (WED).

\section{Introduction}

Over the past few years, the upgrade of resources and processes accompanying the environment around new pathways in terms of sustainable chemistry has become a focal point of the chemical research process. Hence, the development and implementation of chemical processes along with replacing or modifying old processes with new methodologies creates a new direction towards "green chemistry" through the use of natural resources associated with the environment or natural raw materials [1-3]. In recent years, benign and Among the MCRs, the most important MCRS is nitrogen synthesis containing dihydropyridine isotopes. The importance of these compounds lies in the fact that they possess the basic unit of various natural products such as nucleic acids, alkalis, purines, histidine, histamine, along with various pharmacological features such as calcium channel modifiers such as ephedrine, antifungal, anti-tumor, anti-HIV [4-7]. Human immunity, the classical hypothesis of the aforementioned derivatives was carried out under harsh conditions, where the reaction was catalyzed in the presence of formaldehyde in ammonia or Bronsted / Lewis's acids [8-10]. Subsequently, several dihydropyridine synthesis methods were reported, which were developed either by some solvents [11-25].Hazardous ecological synthetic methodologies for multicomponent reactions have received significant attention

$1 *$ Corresponding Author: Email abdulfatahsaifan123@gmail.com 
In this protocol " water extract of dragon fruit( WED ) " is select as an acid catalyst without using dangerous organic reaction medium, transition metal catalyst, toxic solvents / reagents, To the best of our knowledge, there are no earlier reports of WED used as an acid promoter for nitrogen containing Heterocyclic adducts. the extract of dragon fruit (scientific name : cacti ; family : cactaceae ; gen : selenicereus ; Binomial name: common name : dragon) is prepared by first took the peel of dragon fruit and dry it and then crushed by using mixer then took $10 \mathrm{~g}$ of dragon fruit in $100 \mathrm{ml}$ of water and then heat it at $\left(80^{\circ} \mathrm{C}\right)$ and filter it and got water extract of dragon fruit (WED) acid $4.8 \mathrm{pH}$ (as in the fig 1 ).

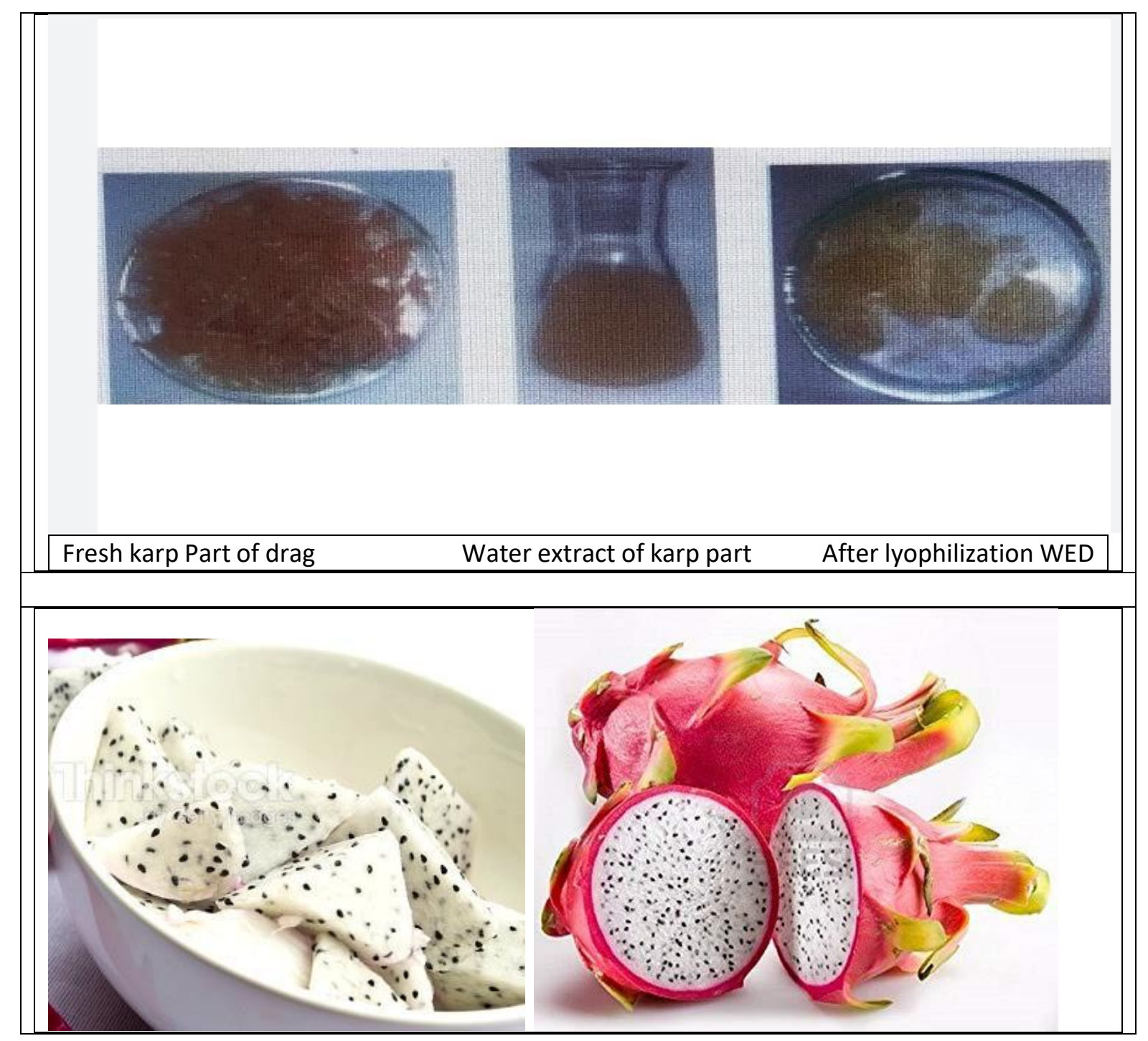

Fig $\{1\}$ This fruit (dragon fruit) is low in calories. It contains different minerals and vitamins. One of them, equivalent to $227 \mathrm{~g}$ contains 136 calories. $29 \mathrm{~g}$ of carbohydrates and $7 \mathrm{~g}$ of dietary fiber. 


\section{Results and Discussion}

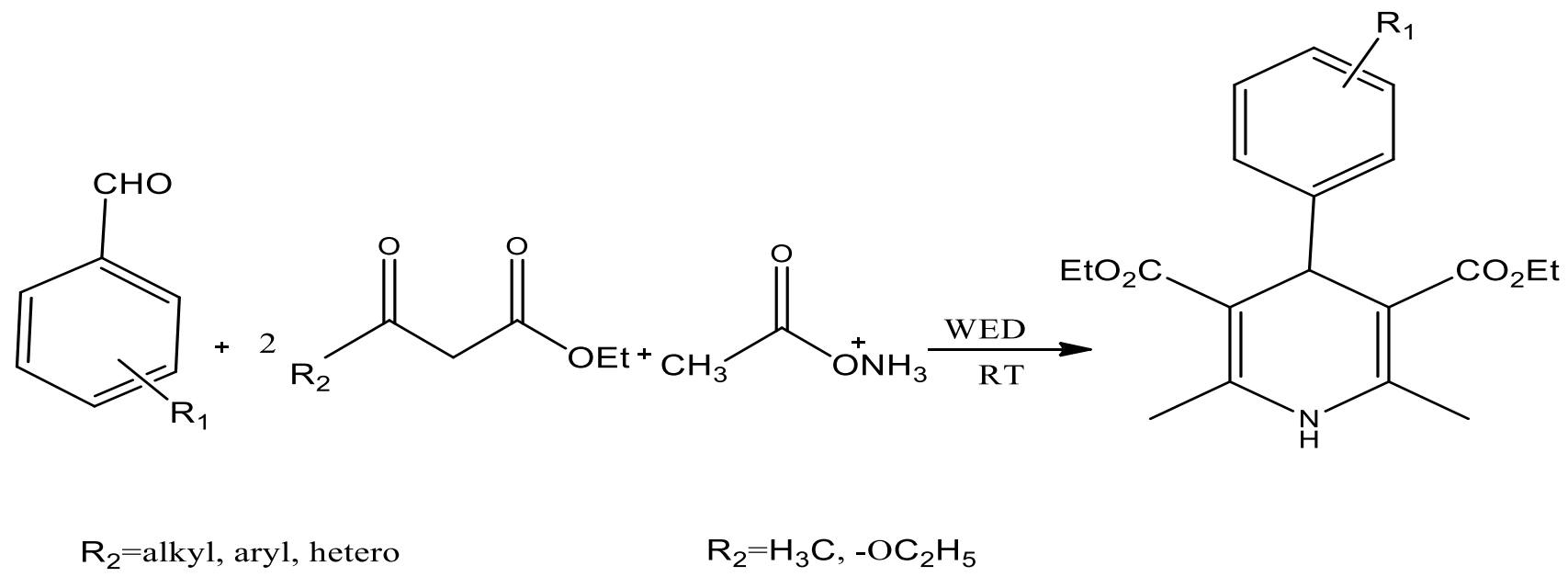

Scheme 1. Preparation of dihydropyridine derivatives.

Our main effort is to develop a greener and efficient synthetic route for effortless conversion of dihydropyridine at room temperature. To analyze the optimum reaction conditions for our suggested reactions, the ability of the catalytic condition was appraised. To finalize this synthetic route and study the effect of the amount of (WED), In this protocol " water extract of dragon fruit( WED ) " is select as an acid catalyst without using dangerous organic reaction medium , transition metal catalyst , toxic solvents / reagents, To the best of our knowledge, there are no earlier reports of (WED) used as an acid promoter for nitrogen containing Heterocyclic adducts. the extract of dragon fruit (scientific name : cacti ; family : cactaceae ; gen : selenicereus ; Binomial name: common name : dragon) is prepared by first took the peel of dragon fruit and dry it and then crushed by using mixer then took $10 \mathrm{~g}$ of dragon fruit in $100 \mathrm{ml}$ of water and then heat it at $\left(80^{\circ} \mathrm{C}\right)$ and filter it and got water extract of dragon fruit (WED) acid $4.8 \mathrm{pH}$ we have done the best method used to prepare dihydropyridine by used of dragon fruit as a catalyst is another example to prepare its derivatives as shown in (scheme 1). We have explored the substrate scope by using different kinds of substituted aldehydes 1 .ethyl acetoacetate 2 and ammonium acetate 3, were condensed together to get desired dihydropyridine derivatives (scheme 1). initially, commercially feasible various substituted aldehydes were allowed to react with ethyl acetoacetate and in a ratio of 1:2:1 ammonium acetate respectively under aforementioned condition (scheme 1). Significant yield of the desired products was obtained, even when the reaction was performed using several substrates containing electron-withdrawing or electron-donating groups on aromatic ring of the aldehyde. It is noteworthy to see that aldehydes which contained halogens as functional groups on the aromatic ring showed smooth conversion to dihydropyridine derivatives (scheme 1; 4aa, 4ag ). However, when heterocyclic aldehydes were used in the same reaction condition, same amount of conversion of dihydropyridine was observed .Further, the desired dihydropyridine products were obtained when acetyl acetone was replaced by ethyl acetoacetate and allowed to react with ammonium acetate and the promoter was found to be highly active, affording up to $92 \%$ of isolated yield. In order to establish more favorable reaction conditions, we thought, there should be 
more scope for further innovation towards milder reaction conditions using different cactus fruit source as a promoter. Meanwhile, we observed that almost all cactus fruit extracts afforded outstanding conversion for both the title reactions. Thus, we have completed these MCRs using these fruit extracts separately under the same reaction environment (scheme 2). Subsequently, in order to figure out other catalytic conditions, we further performed our standard reactions (Scheme 1) with different fruit cactus source (WED-1 and WED-2). Interestingly, similar product conversion was obtained by employing same amount of these promoter to the optimized reaction condition. Indeed, an impressive aspect of this suggested synthetic route is the stability and tolerance of the various functional groups viz., hydroxyl, methoxy, nitro, halides, and heterocycles. We are quite fascinated to see that; similar significant results were obtained when other water extract of fruit promoters (WED-1 and WED-2) were used instead of WED in the reactions.

\section{Conclusion}

In conclusion, we have developed a novel route of dragon fruit extract promoted an efficient, inexpensive and eco-friendly one pot multi component reactions for formulation of highly biologically active dihydropyridine derivatives. This approach is metal as well as solvent free and devoid of any extra additives, co-catalyst, harsh conditions to generate resulting products in high yields. Subsequent studies were successfully performed at room temperature by exploring different substituents through our greener synthetic protocol. Utilizing this kind of natural feedstock in organic transformations would be more decisive and immensely favorable to academic and industrial research. 


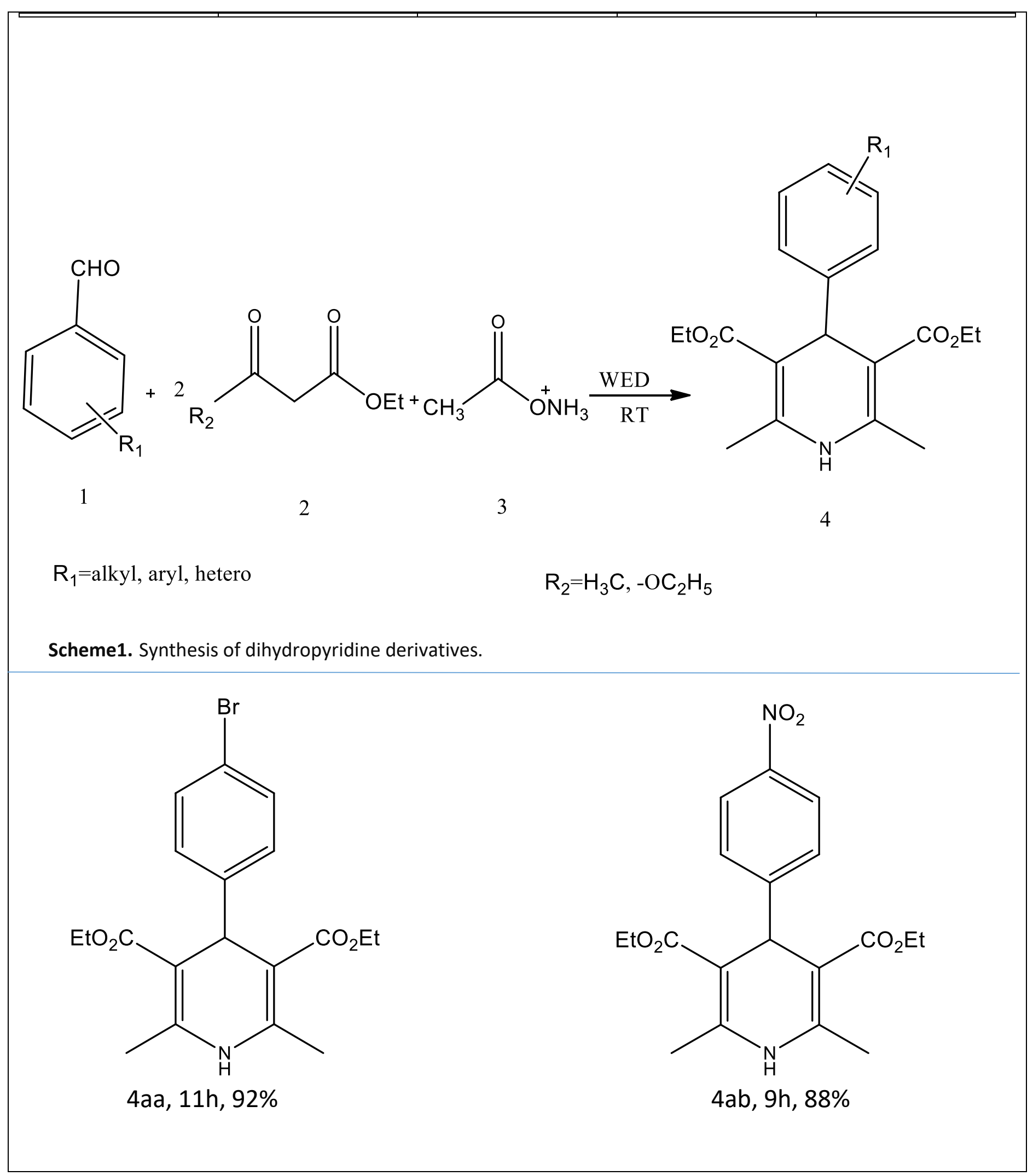




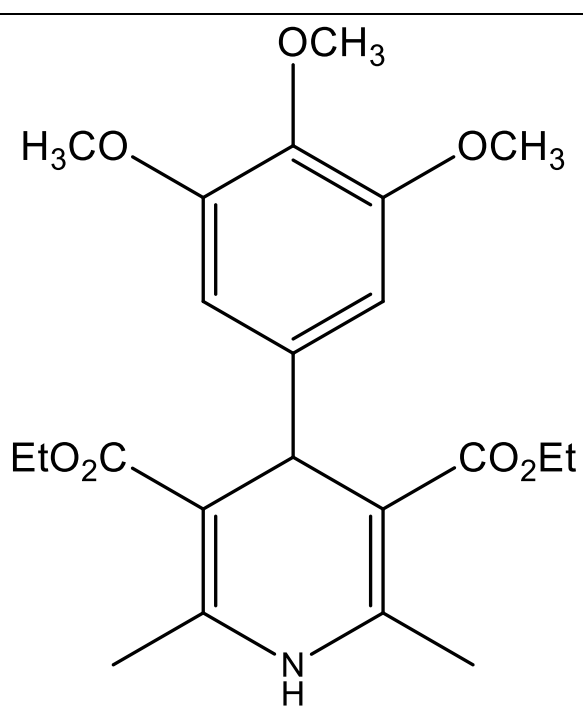

4ac, 8 h, $87 \%$

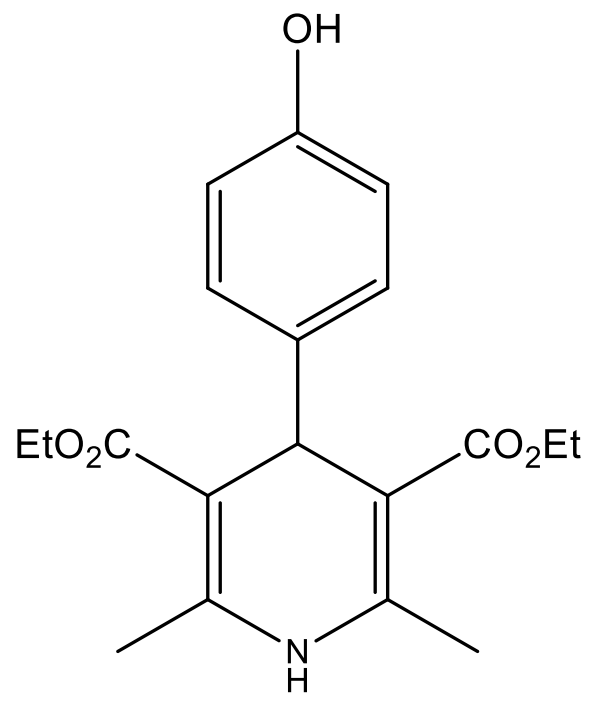

4ae, $10 \mathrm{~h}, 77 \%$

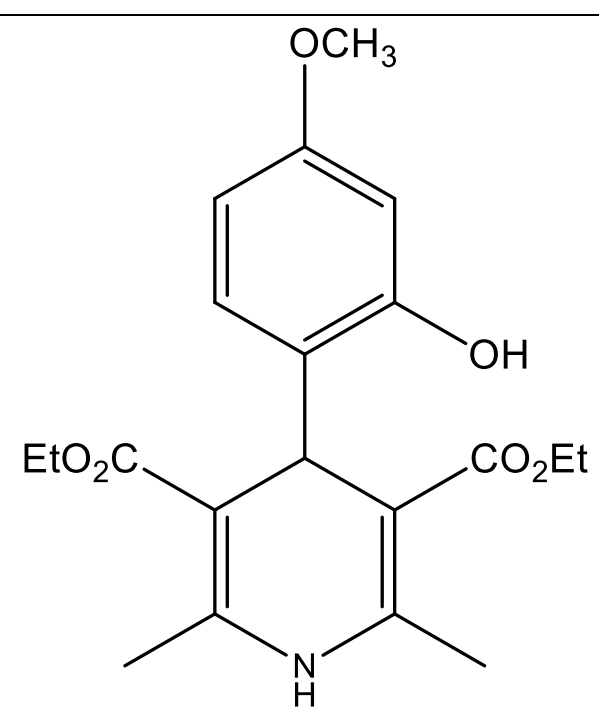

4ad, $12 \mathrm{~h}, 84 \%$

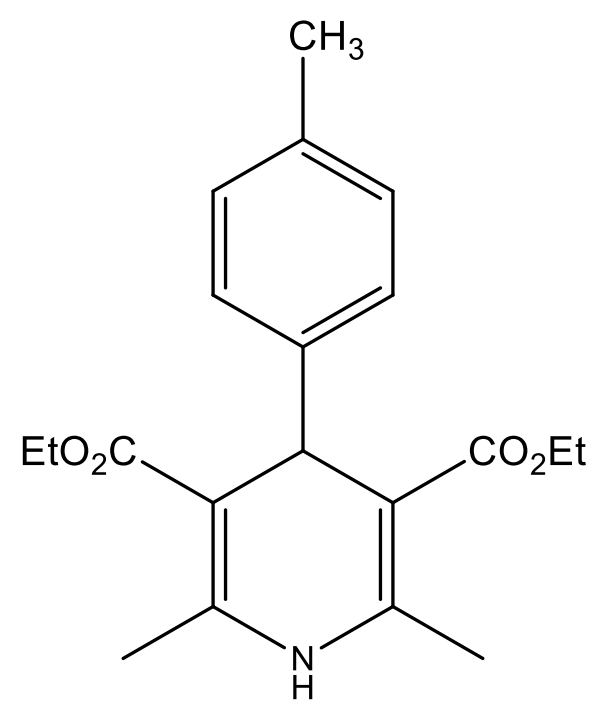

4af, $10 \mathrm{~h}, 78 \%$ 
<smiles>CCOC(=O)C1=C(C)NC(C)=C(C(=O)OCC)C1c1ccc(Cl)cc1</smiles>

4ag, 12h, 91\%<smiles>CCOC(=O)C1=C(C)NC(C)=C(C(=O)OCC)C1c1ccc(O)cc1</smiles>

4ai, 11h, 68\%<smiles>CCOC(=O)C1=C(C)NC(C)=C(C(=O)OCC)C1c1ccc(OC)cc1</smiles>

4ah, $12 \mathrm{~h}, 72 \%$<smiles>CCOC(=O)C1=C(C)NC(C)=C(C(=O)OCC)C1c1cccc([N+](=O)[O-])c1</smiles>

4aj, 11h, 60\%

WED catalyzed cycle-condensation for synthesis of dihydropyridine derivatives[a] Reaction conditions: aldehyde $(1 \mathrm{mmol})$, ethyl acetoacetate $(2 \mathrm{mmol})$, ammonium acetate $(1 \mathrm{mmol})$, promoter $(100 \mathrm{mg})$ at room temperature. All yields are isolated after recrystallization from ethanol.

Different sources of dragon fruit are used to prepare dihydropyridine and its derivatives in order to create more towards more reaction conditions using different sources of cactus fruit as a catalyst. At the same time, we note that all of the fruit extracts of the dragon fruit provided a remarkable transformation for each of the reactions. Therefore, we completed MCRs using fruit extracts separately under the same reaction environment as well as knowledge of other catalytic conditions. We also 
performed reactions with a different source of cactus fruit (WED-1 EWD-2). Interestingly, the conversion of a similar product was obtained by using the same amount of reaction, Similar significant results were obtained when another water extract of fruit promoter (WED-1and WED-2) were used instead of WED in the reaction. As shown below.

\begin{tabular}{|cccc}
\hline $\begin{array}{c}\text { Different dragon fruits source used in the preparation of dihydropyridine and deriva } \\
\text { Entry }\end{array}$ promoter code & fruit source & species scientific name \\
\hline 1 & WED & dragon fruit & Hylocereus undatus \\
2 & WED-1 & dragon fruit & Hylocereus costaricensis \\
3 & WED-2 & dragon fruit & Hylocereus megalanthus
\end{tabular}

(Scheme 2) by using different dragon fruits source, it gives as same the results and discussion as (scheme 1)

\section{Conflict of interest}

The authors declare no conflict of interest

\section{References}

[1] Sheldon, R. A. (1997). Catalysis: the key to waste minimization. Journal of Chemical Technology \& Biotechnology: International Research in Process, Environmental AND Clean Technology, 68(4), 381-388.

[2] Boruah, P. R., Ali, A. A., Saikia, B., Sarma, D., Green Chem. ( 2015), A novel green protocol for ligand free Suzuki-Miyaura cross-coupling reactions in WEB at room temperature. 17, 1442 1445 . .

[3] Okkerse, C., \& Van Bekkum, H. (1999). From fossil to green. Green Chemistry, 1(2), 107-114.

[4] Molina, P. A., Tarraga, Oton, F., Org. Biomol. Chem. (2012), dihydropyridine derivatives: A comprehensive survey of their recognition properties. 10, 1711-1724.

[5] Folkers, K., Harwood, H. J., \& Johnson, T. B. (1932). Researches on pyrimidines. Cxxx. Synthesis of 2-keto-1, 2, 3, 4-tetrahydropyrimidines. Journal of the American Chemical Society, 54(9), 37513758.

[6] Pathal, P., Chem, J. and Pharm. Res. (2014), Synthesis, Characterization and In Vitro Antimicrobial Evaluations of Bis-1, 4-dihydropyrimidines. 6, 838-842.

[7] Shalini, K., Sharma, P. K., \& Kumar, N. (2010). Dihydropyridine and its biological activities: A review. Der Chemica Sinica, 1(3), 36-47.

[8] Timaa, ff. de F. Bragaa, T. C. Netoa, L. da S. Terraa, B. S. Oliveiraa, B. G. F. da Silvaa, D. L Modolob, L. V., Adv. J., of Res. (2015), A mini-review on Biginelli adducts with notable pharmacological properties. 6, 363-373.

[9] Biginelli, P., Gazz. Chim. Ital. (1893), Current Developments in Michael Addition-Based Multicomponent Domino Reactions Involving 1, 3-Dicarbonyls and Derivatives. 23, 360-413.

[10] Kappe, C. O., Tetrahedron (1993), 100 years of the biginelli dihydropyrimidine synthesis. 49, 6937-6946. 
[11] Diao, X., Wang, Y., Jiang, Y., Ma, D., Org. J. Chem. (2009), Assembly of Substituted $1 H$ Benzimidazoles and 1,3-Dihydrobenzimidazol-2-ones via CuI/l-Proline Catalyzed Coupling of Aqueous Ammonia with 2-Iodoacetanilides and 2-Iodophenylcarbamates.74, 7974-7977.

[12] Tlahuext Aca, A., Hern ndez-Fajardo, O., Arevalo, A. Garcia, J. J., Dalton Trans. (2014), Synthesis of tetra-substituted dihydropyridine and 2- dihydropyridine by $\mathrm{Ni}(0)$-catalyzed dehydrogenation of benzylic-type imines. 43, 15997-16005.

[13] Guru, M. M. Ali, M. A. Punniyamurthy, T. J. Org. Chem. (2011), Copper-Mediated Synthesis of Substituted 2-Aryl- $N$-benzylbenzimidazoles and 2-Arylbenzoxazoles via $\mathrm{C}-\mathrm{H}$ Functionalization/C-N/C-O Bond Formation. 76, 5295- 5308.

[14] Ramesh, K., Murthy, S. N., Karnakar, K., Nageswar, Y. V. D., Vijayalakhshmi, K., Devi, B. L. P., \& Prasad, R. B. N. (2012). A novel bioglycerol-based recyclable carbon catalyst for an efficient one-pot synthesis of highly substituted imidazoles. Tetrahedron Letters, 53(9), 1126-1129.

[15] Usyatinsky A. Y., Khmelnitsky, Y. L., Tetrahedron Lett. (2000), Microwave-Assisted, SolventFree and Parallel Synthesis of Some Novel Substituted dihydropyridine of Biological Interest. 41, 5031- 5034.

[16] Samanta, S., Roy, D., Khamarui, S. Maiti, D. K. Chem. Commun. (2014), Ni (ii)-salt catalyzed activation of primary amine-sp ${ }^{3} \mathrm{C}_{\alpha}-\mathrm{H}$ and cyclization with 1,2-diketone to tetrasubstituted dehydrogenation. 50, 2477-2480.

[17] Heravi, M. M. Derikv, F. Haghighi, M. Monatsh. Chem. (2008), Lewis acid catalyst free synthesis of substituted dihydropyridine in 2,2,2-trifluoroethanol. 139, 31-33.

[18] Chawla, A., Sharma, A., \& kumar Sharma, A. (2012). A convenient approach for the synthesis of imidazole derivatives using microwaves. Synthesis, 5(6), 7.

[19] Nagarajaiah, H., Mukhopadhyay, A., \& Moorthy, J. N. (2016). Biginelli reaction: an overview. Tetrahedron Letters, 57(47), 5135-5149.

[20] Bai, J. Lu, Y. Synthesis (2002), Catalysis of the Biginelli Reaction by Ferric and Nickel Chloride Hexahydrates. One-Pot Synthesis of 3, 4- dihydropyridine -2(1H)-ones. 4, 466-470.

[21] Dondoni, A. Massi, A. Sabbatini, S. TetrahedronLett. (2002), A Novel Catalyst for One-Pot Synthesis of Substituted 3,4-Dihydropyrimidin-2-(1H)-ones via Biginelli Reaction Under SolventFree Conditions. 43, 5913- 5916.

[22] Ma, Y., Qian, C., Wang, L., \& Yang, M. (2000). Lanthanide triflate catalyzed Biginelli reaction. Onepot synthesis of dihydropyrimidinones under solvent-free conditions. The Journal of organic chemistry, 65(12), 3864-3868.

[23] Khademinia, S., Behzad, M., Jahromi, H. S., RSC Adv. (2015), Solid state synthesis, characterization, optical properties and cooperative catalytic performance of bismuth vanadate nanocatalyst for Biginelli reactions. 5, 24313- 24318.

[24] Lopez-Pestana, J. M., Avila-Rey, M. J., \& Martin-Aranda, R. M. (2002). Ultrasound-promoted Nalkylation of imidazole. Catalysis by solid-base, alkali-metal doped carbons. Green Chemistry, 4(6), 628-630.

[25] Qu, G. R., Zhang, H. L., Niu, H. Y., Xue, Z. K., Lv, X. X., \& Guo, H. M. (2012). Synthesis of C6azolyl purine nucleosides via $\mathrm{C}-\mathrm{N}$ coupling reaction of unprotected 6-chloropurine nucleosides and N-heterocycles under catalyst-and solvent-free conditions. Green Chemistry, 14(7), 1877-1879. 